\title{
Novel Hyperbolic Homoclinic Solutions of the Helmholtz-Duffing Oscillators
}

\author{
Yang-Yang Chen, ${ }^{1}$ Shu-Hui Chen, ${ }^{2}$ and Wei-Wei Wang ${ }^{2}$ \\ ${ }^{1}$ Key Laboratory of Vibration Control and Structural Safety, Guangzhou University, Guangzhou 510405, China \\ ${ }^{2}$ Department of Applied Mechanics and Engineering, Sun Yat-sen University, Guangzhou 510275, China \\ Correspondence should be addressed to Yang-Yang Chen; yychen@gzhu.edu.cn
}

Received 28 May 2015; Revised 23 August 2015; Accepted 26 August 2015

Academic Editor: Chia-Ming Chang

Copyright (c) 2016 Yang-Yang Chen et al. This is an open access article distributed under the Creative Commons Attribution License, which permits unrestricted use, distribution, and reproduction in any medium, provided the original work is properly cited.

\begin{abstract}
The exact and explicit homoclinic solution of the undamped Helmholtz-Duffing oscillator is derived by a presented hyperbolic function balance procedure. The homoclinic solution of the self-excited Helmholtz-Duffing oscillator can also be obtained by an extended hyperbolic perturbation method. The application of the present homoclinic solutions to the chaos prediction of the nonautonomous Helmholtz-Duffing oscillator is performed. Effectiveness and advantage of the present solutions are shown by comparisons.
\end{abstract}

\section{Introduction}

It has been widely accepted that homoclinic solutions play a fundamental role in global bifurcations and chaos predictions of dynamical systems $[1,2]$. For instance, the experimental study of certain magnetic pendulum verified the homoclinic solutions as the precursors to chaotic vibration [3]. Some occurrences of homoclinic solutions can be regarded as the criterion from single well chaos to cross well chaos motion of oscillators [4], or as the onsets of chaotic vibrations of asymmetric nonconservative oscillators [5]. Homoclinic solutions were also adopted in bifurcation and chaotic vibration controls for beam structures [6,7]. Another typical application of homoclinic solutions aims at solitary wave studies. For instance, a proper homoclinic solution can govern the solitary roll waves down an open inclined channel [8], or optical solitary waves propagating in fibers $[9,10]$. The association between the singular solitary waves and homoclinic solutions can be interpreted based on phase plane analysis [11].

Because of their importance in nonlinear systems, many homoclinic solutions have been derived in the past few decades. Such works include but are not limited to the following: Xu et al. [12] proposed the perturbation-incremental method for homoclinic solutions; Chan et al. [13] applied the perturbation-incremental method to study the stability and the homoclinic bifurcations of limit cycles; Belhaq et al. [14] analytically developed criterions for predicting homoclinic connection of limit cycle. Mikhlin and Manucharyan [15] and Manucharyan and Mikhlin [16] applied the Padé and quasiPadé approximants for homo- and heteroclinic solutions. Y. Y. Chen and S. H. Chen [17] and Chen et al. [18] developed perturbation techniques by hyperbolic functions for homoclinic solutions of strongly nonlinear oscillators. Cao et al. [19] improved the perturbation-incremental homoclinic solutions for strongly nonlinear oscillators. Recently, Li et al. [20] improved the perturbation method based on harmonic functions to derive homoclinic solutions of HelmholtzDuffing oscillators.

Nevertheless, to the best of our knowledge, the completely analytical, exact, and explicit homoclinic solution of the strongly nonlinear Helmholtz-Duffing oscillators has not yet been derived, in spite of the wide application of its equation for many engineering problems such as ship dynamics, oscillation of the human ear drum, oscillations of onedimensional structural system with an initial curvature, some electrical circuits, microperforated panel absorber, and heavy symmetric gyroscope [21-29]. It should be pointed out that the previous typical solutions [14-18] become invalid for such mix-parity systems. Even for the conservative HelmholtzDuffing oscillator, solutions by the perturbation methods [12, 
$13,19,20$ ] based on generalized harmonic functions can only be obtained implicitly, in which the infinite time domain of a homoclinic motion has to be transformed into a finite period of the harmonic. Moreover, for strongly nonlinear oscillators, as the perturbation-incremental method $[12,13,19]$ consists of perturbation procedure with the incremental harmonic balance method, their solutions are always expressed by harmonic functions with numerical coefficients. That means such implicit solutions are semianalytical and seminumerical and cumbersome for practical application.

This paper aims to present new homoclinic solutions of the Helmholtz-Duffing oscillators. The completely analytical, exact, and explicit homoclinic solution of the conservative Helmholtz-Duffing oscillator will be derived by a hyperbolic function balance procedure. Then the homoclinic solution of the self-excited Helmholtz-Duffing oscillator will also be obtained by an extended hyperbolic perturbation method. The application of the present solutions to the chaos prediction of the nonautonomous Helmholtz-Duffing oscillator is performed. The preference of the present solution will be illustrated by comparison.

\section{The Explicit and Exact Homoclinic Solution of the Undamped Helmholtz-Duffing Oscillator}

Consider the homoclinic solution of the undamped Helmholtz-Duffing equation

$$
\ddot{x}+c_{1} x+c_{2} x^{2}+c_{3} x^{3}=0 .
$$

If $c_{2}=0$, (1) becomes the classical Duffing equation, which possesses a homoclinic solution with $c_{1}<0$ and $c_{3}>0$. Such homoclinic solution of classical Duffing equation has been discussed in detail in [17], in which the solution can be written as

$$
x_{0}= \pm \sqrt{\frac{-2 c_{1}}{c_{3}}} \operatorname{sech} \sqrt{-c_{1}} t
$$

If $c_{3}=0$, (1) becomes the classical Helmholtz equation, which possesses a homoclinic solution. Such homoclinic solution of classical Helmholtz equation has been discussed in detail in [18], in which the solution can be written as

$$
x_{0}=\frac{3\left|c_{1}\right|}{2 c_{2}} \operatorname{sech}^{2} \frac{\sqrt{\left|c_{1}\right|}}{2} t-\frac{\left(\left|c_{1}\right|+c_{1}\right)}{2 c_{2}} \text {, }
$$

Noting the relationship as below,

$$
\operatorname{sech}^{2} \frac{\sqrt{\left|c_{1}\right|}}{2} t=\frac{1}{\cosh ^{2}\left(\sqrt{\left|c_{1}\right|} / 2\right) t}=\frac{2}{1+\cosh \sqrt{\left|c_{1}\right|} t}
$$

we can rewrite (2) and (3), respectively; that is,

$$
\begin{aligned}
& x_{0}=\frac{3\left|c_{1}\right|}{c_{2}} \frac{1}{\left(1+\cosh \sqrt{\left|c_{1}\right|} t\right)}-\frac{\left(\left|c_{1}\right|+c_{1}\right)}{2 c_{2}}, \\
& x_{0}= \pm \sqrt{\frac{-2 c_{1}}{c_{3}}} \frac{1}{\cosh \sqrt{\left|c_{1}\right|}} .
\end{aligned}
$$

Here, to find a proper trial solution form for (1), we can observe the two special cases above. It can be seen that the two expressions above are similar, because they have the common form expressed as

$$
x_{0}=\frac{a_{0}}{b+\cosh \omega_{0} t}+u,
$$

in which, when $c_{3}=0$, the constants of (6) are $a_{0}=3\left|c_{1}\right| / c_{2}$, $b=1, \omega_{0}=\sqrt{c_{1}}$, and $u=-\left(\left|c_{1}\right|+c_{1}\right) / 2 c_{2}$. While when $c_{2}=0$, $c_{1}<0$, and $c_{3}>0$, the constants of (6) are $a_{0}= \pm \sqrt{-2 c_{1} / c_{3}}$, $b=0, \omega_{0}=\sqrt{c_{1}}$, and $u=0$.

Thus, the time derivative of (6) is

$$
\dot{x}_{0}=-\frac{a_{0} \omega_{0} \sinh \omega_{0} t}{\left(b+\cosh \omega_{0} t\right)^{2}} .
$$

Note that $(u, 0)$ is the homoclinic point. For $c_{2} \neq 0$ and $c_{3} \neq 0$, we adopt (6) as a trial solution for the homoclinic solution of (1) and try to determine all its constants by substituting (6) into (1); that is,

$$
\begin{aligned}
& {\left[( b + \operatorname { c o s h } \omega _ { 0 } t ) ^ { - 3 } \left(c_{1} a_{0} b^{2}+c_{1} b^{3} u+c_{2} b^{3} u^{2}+c_{2} a_{0}^{2} b\right.\right.} \\
& \quad+2 c_{2} a_{0} b^{2} u+c_{3} b^{3} u^{3}+c_{3} a_{0}^{2}+3 c_{3} a_{0} b^{2} u^{2}+3 c_{3} a_{0}^{2} b u \\
& \left.\quad-2 a_{0} \omega_{0}^{2}\right)+\left(b+\cosh \omega_{0} t\right)^{-2}\left(2 c_{1} a_{0} b+3 c_{1} b^{2} u\right. \\
& \quad+c_{2} a_{0}^{2}+4 c_{2} a_{0} b u+3 c_{2} b^{2} u^{2}+3 c_{3} a_{0}^{2} u+6 c_{3} a_{0} b u^{2} \\
& \left.\quad+3 c_{3} b^{2} u^{3}-a_{0} b \omega_{0}^{2}\right)+\left(b+\cosh \omega_{0} t\right)^{-1}\left(c_{1} a_{0}\right. \\
& \quad+3 c_{1} b u+2 c_{2} a_{0} u+3 c_{2} b u^{2}+3 c_{3} a_{0} u^{2}+3 c_{3} b u^{3} \\
& \left.\left.+a_{0} \omega_{0}^{2}\right)+\left(c_{1} u+c_{2} u^{2}+c_{3} u^{3}\right)\right]=0 .
\end{aligned}
$$

In order to balance (8) for all time $t$, we equate coefficients of like powers of the hyperbolic function term $\left(b+\cosh \omega_{0} t\right)$ and get the following nonlinear algebraic equations:

$$
\begin{aligned}
& c_{1} a_{0} b^{2}+c_{1} b^{3} u+c_{2} b^{3} u^{2}+c_{2} a_{0}^{2} b+2 c_{2} a_{0} b^{2} u+c_{3} b^{3} u^{3} \\
& \quad+c_{3} a_{0}^{2}+3 c_{3} a_{0} b^{2} u^{2}+3 c_{3} a_{0}^{2} b u-2 a_{0} \omega_{0}^{2}=0, \\
& 2 c_{1} a_{0} b+3 c_{1} b^{2} u+c_{2} a_{0}^{2}+4 c_{2} a_{0} b u+3 c_{2} b^{2} u^{2}+3 c_{3} a_{0}^{2} u \\
& \quad+6 c_{3} a_{0} b u^{2}+3 c_{3} b^{2} u^{3}-a_{0} b \omega_{0}^{2}=0, \\
& c_{1} a_{0}+3 c_{1} b u+2 c_{2} a_{0} u+3 c_{2} b u^{2}+3 c_{3} a_{0} u^{2}+3 c_{3} b u^{3} \\
& \quad+a_{0} \omega_{0}^{2}=0, \\
& c_{1} u+c_{2} u^{2}+c_{3} u^{3}=0 .
\end{aligned}
$$


From (12),

$$
u=0
$$

or

$$
u=\frac{-c_{2} \pm \sqrt{c_{2}^{2}-4 c_{1} c_{3}}}{2 c_{3}}
$$

The left hand side of (12) can be regarded as the restoring force of the oscillator with $x=u$. In other words, (12) means that the displacement derivative of the potential energy curve at $x=u$ is zero. Furthermore, we have to make sure that the potential energy curve at $x=u$ is not concave. Thus, the displacement derivative of the restoring force at $x=u$ will not be positive; that is,

$$
\left.\frac{d}{d x}\left(c_{1} x+c_{2} x^{2}+c_{3} x^{3}\right)\right|_{x=u}=c_{1}+2 c_{2} u+3 c_{3} x^{2} \leq 0 .
$$

Therefore, $u$ can be determined by (13)-(15) and then, (9)-(11) can be discussed, respectively, in the two cases as follows.

Case $1(u=0)$. Equations (9)-(11) can be rewritten as below

$$
\begin{aligned}
c_{1} b^{2}+c_{2} a_{0} b+c_{3} a_{0}^{2}-2 \omega_{0}^{2} & =0, \\
2 c_{1} b+c_{2} a_{0}-b \omega_{0}^{2} & =0, \\
c_{1}+\omega_{0}^{2} & =0,
\end{aligned}
$$

by which one can obtain

$$
\begin{aligned}
& \omega_{0}=\sqrt{-c_{1}}, \\
& a_{0}= \pm \frac{3 \sqrt{2} c_{1}}{\sqrt{2 c_{2}^{2}-9 c_{1} c_{3}}}, \\
& b=\mp \frac{\sqrt{2} c_{2}}{\sqrt{2 c_{2}^{2}-9 c_{1} c_{3}}} .
\end{aligned}
$$

Case $2\left(u=\left(-c_{2} \pm \sqrt{c_{2}^{2}-4 c_{1} c_{3}}\right) / 2 c_{3}\right)$. As $a_{0}$ and $u$ are nonzero, multiplying (12) by $-b^{2}\left(b+a_{0} / u\right)$ and then adding it to (9) yield

$$
c_{2} a_{0} b+c_{2} b^{2} u+c_{3} a_{0}^{2}+3 c_{3} a_{0} b u+2 c_{3} b^{2} u^{2}-2 \omega_{0}^{2}=0 .
$$

Multiplying (12) by $-b\left(3 b+2 a_{0} / u\right)$ and then adding it to (10), the latter becomes

$$
c_{2} a+2 c_{2} b u+3 c_{3} a u+4 c_{3} b u^{2}-b \omega_{0}^{2}=0 .
$$

Multiplying (12) by $\left(-3 b+a_{0} / u\right)$ and then adding it to (11), we also get

$$
c_{2} u+2 c_{3} u^{2}+\omega_{0}^{2}=0
$$

Thus from (18)-(20),

$$
\begin{aligned}
& \omega_{0}=\sqrt{-u\left(c_{2}+2 c_{3} u\right)}, \\
& a_{0}= \pm \frac{3 \sqrt{2} u\left(c_{2}+2 c_{3} u\right)}{\sqrt{c_{2}\left(2 c_{2}+3 c_{3} u\right)}} \\
& b=\mp \frac{\sqrt{2}\left(c_{2}+3 c_{3} u\right)}{\sqrt{c_{2}\left(2 c_{2}+3 c_{3} u\right)}} .
\end{aligned}
$$

Furthermore, noting that, for the homoclinic solution, the potential energy values at $t=0$ and at $t= \pm \infty$ should be equal, we have

$$
\begin{aligned}
& \frac{1}{2} c_{1} u^{2}+\frac{1}{3} c_{2} u^{3}+\frac{1}{4} c_{3} u^{4} \\
& =\frac{1}{2} c_{1}\left(\frac{a_{0}}{b+1}+u\right)^{2}+\frac{1}{3} c_{2}\left(\frac{a_{0}}{b+1}+u\right)^{3}+\frac{1}{4} c_{3}\left(\frac{a_{0}}{b+1}+u\right)^{4},
\end{aligned}
$$

by which the final values of $a_{0}, b$, and $\omega_{0}$ expressed by (17) or (21) can be selected.

Example 1. Here we apply the method for equation

$$
\ddot{x}-x-3 x^{2}+x^{3}=0,
$$

which is a case of (1) with $c_{1}=-1, c_{2}=-3$, and $c_{3}=1$. From (14), (21), and (22), we can determine all the constants and get the homoclinic solution as

$$
\begin{aligned}
& x=\frac{-1}{1 \pm \sqrt{3 / 2} \cosh t}, \\
& \dot{x}=\frac{\sinh t}{ \pm \sqrt{2 / 3}+2 \cosh t \pm \sqrt{3 / 2} \cosh ^{2} t} .
\end{aligned}
$$

The time histories and the phase portraits of the solutions by different methods are shown in Figures 1 and 2, respectively. It can be seen from the figures that the present method yields accurate and explicit solutions in both the figures, while the generalized harmonic function perturbation method can only provide valid solution in Figure 2 . The reason is that based on harmonic functions $[12,13,19,20]$ the homoclinic solutions can only be expressed implicitly by the nonlinear time scale they adopted and be investigated only in phase planes. Such implicit solutions are too abstract or cumbersome to use in some practical problems. Therefore, the present explicit solutions in respect to time $t$ are more applicable.

\section{Perturbation Homoclinic Solution of the Self-Excited Helmholtz-Duffing Oscillator}

Consider the homoclinic solution of the self-excited Helmholtz equation

$$
\ddot{x}+c_{1} x+c_{2} x^{2}+c_{3} x^{3}=\varepsilon f(\mu, x, \dot{x}),
$$




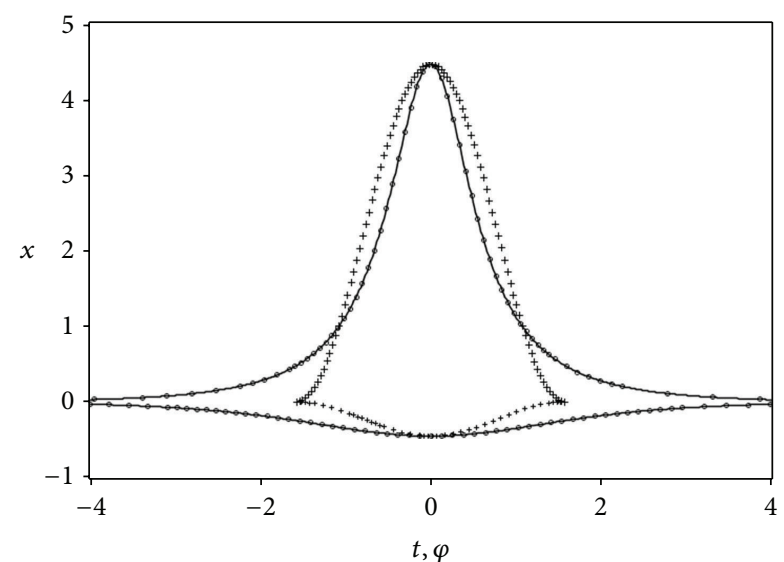

FIGURE 1: Time histories for homoclinic solutions of (23): - AUTO numerical method [30,31]; $\bigcirc \bigcirc \bigcirc$ present method; +++ generalized harmonic function perturbation method [20].

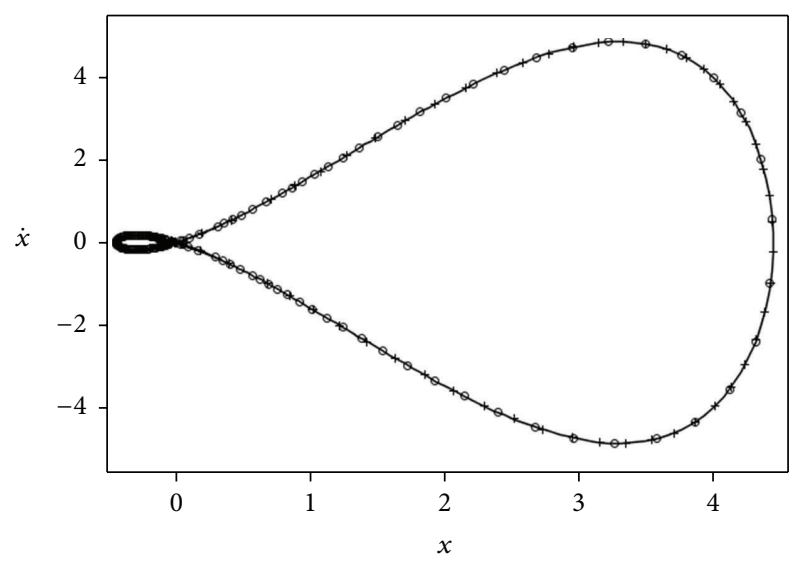

FIGURE 2: Phase portrait for homoclinic solutions $x$ of (23): AUTO numerical method [30, 31]; $\bigcirc \bigcirc \bigcirc$ present method; +++ generalized harmonic function perturbation method [20].

where $\mu$ denotes constants. We assume the homoclinic solution of (26) can still be expressed in the similar form of (6); that is,

$$
x=\frac{a}{b+\cosh \tau}+u
$$

however, the amplitude $a$ and the nonlinear time scale $\tau$ will depend upon the perturbation parameter $\varepsilon$. Thus $a$ and $\omega$, respectively, can be expanded in the powers of $\varepsilon$; that is,

$$
\begin{aligned}
a & =a_{0}+\varepsilon a_{1}+\cdots=\sum_{n=0}^{\infty} \varepsilon^{n} a_{n}, \\
\frac{d \tau}{d t} & =\omega(\tau)=\omega_{0}+\varepsilon \omega_{1}(\tau)+\cdots=\sum_{n=0}^{\infty} \varepsilon^{n} \omega_{n}(\tau) .
\end{aligned}
$$

Then (27) can be rewritten as

$$
x=x_{0}+\varepsilon x_{1}+\cdots=\sum_{n=0}^{\infty} \varepsilon^{n} x_{n},
$$

where

$$
x_{n}=\frac{a_{n}}{b+\cosh \tau} \text {. }
$$

Then

$$
\frac{d x_{n}}{d \tau}=x_{n}^{\prime}=-\frac{a_{n} \sinh \tau}{(b+\cosh \tau)^{2}} .
$$

After substituting (29) and (30) into (26), equating coefficients of like powers of $\varepsilon$ yields the following equations:

$$
\begin{aligned}
& \varepsilon^{0}: \omega_{0}^{2} x_{0}^{\prime \prime}+c_{1} x_{0}+c_{2} x_{0}^{2}+c_{3} x_{0}^{3}=0, \\
& \varepsilon^{1}: \omega_{0}^{2} x_{1}^{\prime \prime}+\omega_{0} \frac{d}{d \tau}\left(\omega_{1} x_{0}^{\prime}\right)+\omega_{1} \frac{d}{d \tau}\left(\omega_{0} x_{0}^{\prime}\right) \\
& \quad+\left(c_{1}+2 c_{2} x_{0}+3 c_{3} x_{0}^{2}\right) x_{1}=f\left(\mu, x_{0}, \omega_{0} x_{0}^{\prime}\right),
\end{aligned}
$$

Then solutions $x_{0}, x_{1}, \ldots$ can be determined by solving linear equations (33), (34), ... one by one. It can be seen that (33) is obtained from (1) via the transformation in (29). Therefore, the homoclinic solution of (33) can be given by (6). Multiplying (34) by $x_{0}^{\prime}$ and integrating it from $\tau_{0}$ to $\tau$, we obtain

$$
\begin{aligned}
\left.\omega_{0} \omega_{1} x_{0}^{\prime 2}\right|_{\tau_{0}} ^{\tau}= & I(\tau)-I\left(\tau_{0}\right)-\left.\omega_{0}^{2} x_{0}^{\prime} x_{1}^{\prime}\right|_{\tau_{0}} ^{\tau} \\
& -\left.x_{1}\left(c_{1} x_{0}+c_{2} x_{0}^{2}+c_{3} x_{0}^{3}\right)\right|_{\tau_{0}} ^{\tau},
\end{aligned}
$$

where

$$
I(\tau)=\int_{0}^{\tau} x_{0}^{\prime} f\left(\mu, x_{0}, \omega_{0} x_{0}^{\prime}\right) d \tau
$$

Noting the properties of hyperbolic functions, we have

$$
\begin{aligned}
x_{0}(0) & =\frac{a_{0}}{(b+1)}+u, \\
x_{0}( \pm \infty) & =u, \\
x_{1}(0) & =\frac{a_{1}}{(b+1)}, \\
x_{1}( \pm \infty) & =x_{0}^{\prime}(0)=x_{0}^{\prime}( \pm \infty)=x_{1}^{\prime}(0)=x_{1}^{\prime}( \pm \infty) \\
& =0 .
\end{aligned}
$$

Thus letting $\tau_{0}=-\infty$ and $\tau=+\infty$ in (35), we derive

$$
\int_{-\infty}^{+\infty} x_{0}^{\prime} f\left(\mu, x_{0}, \omega_{0} x_{0}^{\prime}\right) d \tau=I(+\infty)-I(-\infty)=0 .
$$

Equation (38), which can also be derived by the classical Melnikov method, represents the critical condition under which the homoclinic bifurcation occurs. In other words, there exists a homoclinic solution once all the constants in (26) satisfy (38). Letting $\tau_{0}=0$ and $\tau=+\infty$ in (35) gives 


$$
a_{1}=\frac{-(b+1) I(+\infty)}{c_{1}\left(a_{0} /(b+1)+u\right)+c_{2}\left(a_{0} /(b+1)+u\right)^{2}+c_{3}\left(a_{0} /(b+1)+u\right)^{3}} .
$$

Furthermore, substituting $\tau_{0}=0$ into (35) yields

$$
\begin{aligned}
\omega_{1} & =\frac{1}{\omega_{0} x_{0}^{\prime 2}}\left\{I(\tau)-\omega_{0}^{2} x_{0}^{\prime} x_{1}^{\prime}-x_{1}\left(c_{1} x_{0}+c_{2} x_{0}^{2}\right.\right. \\
& \left.+c_{3} x_{0}^{3}\right)+\left(\frac{a_{1}}{b+1}\right)\left[c_{1}\left(\frac{a_{0}}{b+1}+u\right)\right. \\
& \left.\left.+c_{2}\left(\frac{a_{0}}{b+1}+u\right)^{2}+c_{3}\left(\frac{a_{0}}{b+1}+u\right)^{3}\right]\right\} .
\end{aligned}
$$

The three equations above allow $\mu_{c}, a_{1}$, and $\omega_{1}$ to be determined one by one. As an illustration, here we consider

$$
f\left(\mu, x_{0}, \omega_{0} x_{0}^{\prime}\right)=\omega_{0} x_{0}^{\prime}\left(\mu_{0}+\mu_{1} x_{0}+\mu_{2} x_{0}^{2}\right),
$$

in which $\mu_{0}, \mu_{1}$, and $\mu_{2}$ are constants. Noting (41), and substituting (6) and (7) into (36), the latter becomes

$$
\begin{aligned}
& I(\tau)=\frac{a_{0}^{2} \omega_{0}}{\left(b^{2}-1\right)^{2}}\left\{\frac{\left(-b \mu_{0}+\beta \mu_{1}+\gamma \mu_{2}\right)}{\sqrt{b^{2}-1}}\right. \\
& \cdot \operatorname{arctanh}\left(\sqrt{\frac{b-1}{b+1}} \tanh \left(\frac{\tau}{2}\right)\right) \\
& +\frac{\sinh (\tau)}{6(\cosh (\tau)+b)^{3}}\left[\mu_{0} \sum_{i=0}^{2} A_{i} \cosh ^{i}(\tau)\right. \\
& +\frac{\mu_{1}}{4\left(b^{2}-1\right)(\cosh (\tau)+b)} \sum_{i=0}^{3} B_{i} \cosh ^{i}(\tau) \\
& \left.\left.+\frac{\mu_{2}}{20\left(b^{2}-1\right)^{2}(\cosh (\tau)+b)^{2}} \sum_{i=0}^{4} D_{i} \cosh ^{i}(\tau)\right]\right\},
\end{aligned}
$$

where the expressions of the constants $\beta, \gamma, A_{i}, B_{i}$, and $D_{i}$ are listed in Appendix. Noting that, in (41), $x_{0}$ is an even function and $x_{0}^{\prime}$ is an odd function with respect to $\tau$, (38) can be rewritten as

$$
I(+\infty)=0
$$

Thus substituting (42) into (43), one derives

$$
\begin{aligned}
\mu_{0} A_{2} & +\frac{\mu_{1} B_{3}}{4\left(b^{2}-1\right)}+\frac{\mu_{2} D_{4}}{20\left(b^{2}-1\right)^{2}} \\
& +\frac{6\left(-b \mu_{0}+\beta \mu_{1}+\gamma \mu_{2}\right)}{\sqrt{b^{2}-1}} \operatorname{arctanh}\left(\sqrt{\frac{b-1}{b+1}}\right)
\end{aligned}
$$

$$
=0 .
$$

Equation (44) is the condition under which homoclinic bifurcation occurs. Substituting (43) into (39) gives

$$
a_{1}=0 \text {. }
$$

Then (40) can be rewritten as

$$
\begin{aligned}
& \omega_{1}(\tau)=\frac{(\cosh (\tau)+b)^{4}}{\left(b^{2}-1\right)^{2} \sinh ^{2}(\tau)}\left\{\frac{\left(-b \mu_{0}+\beta \mu_{1}+\gamma \mu_{2}\right)}{\sqrt{b^{2}-1}}\right. \\
& \cdot \operatorname{arctanh}\left(\sqrt{\frac{b-1}{b+1}} \tanh \left(\frac{\tau}{2}\right)\right) \\
& +\frac{\sinh (\tau)}{6(\cosh (\tau)+b)^{3}}\left[\mu_{c} \sum_{i=0}^{2} A_{i} \cosh ^{i}(\tau)\right. \\
& +\frac{\mu_{1}}{4\left(b^{2}-1\right)(\cosh (\tau)+b)} \sum_{i=0}^{3} B_{i} \cosh ^{i}(\tau) \\
& \left.\left.+\frac{\mu_{2}}{20\left(b^{2}-1\right)^{2}(\cosh (\tau)+b)^{2}} \sum_{i=0}^{4} D_{i} \cosh ^{i}(\tau)\right]\right\} .
\end{aligned}
$$

Finally, the expression for homoclinic solution can be expressed by

$$
\begin{aligned}
& x=\frac{a_{0}}{b+\cosh \tau}+u+O\left(\varepsilon^{2}\right), \\
& \dot{x}=-\frac{\left[\omega_{0}+\varepsilon \omega_{1}(\tau)\right] a_{0} \sinh \tau}{(b+\cosh \tau)^{2}}+O\left(\varepsilon^{2}\right) .
\end{aligned}
$$

It can be seen that once $c_{2}$ or $c_{3}$ becomes zero, the present procedures can be reduced to the methods and solutions presented in [17] or in [18].

Example 2. Consider the equation

$$
\ddot{x}+x-x^{2}-2 x^{3}=\left(\mu_{0}-2 x+9 x^{2}\right) \dot{x}
$$

which is a generalized Helmholtz-Duffing-Van der Pol equation with $c_{1}=1, c_{2}=-1, c_{3}=-2, \varepsilon=1, \mu_{1}=-2$, and $\mu_{2}=9$. Here $\mu_{0}$ is the classical damping coefficient which is considered as the bifurcation parameter. A limit cycle motion can break up by homoclinic bifurcation at a critical value of the damping coefficient. From (14), (15), (21), and (22), we have $u=0.5, \omega_{0}=1 / \sqrt{2}, a_{0}=-9 / \sqrt{10}$, and $b=4 \sqrt{2} / \sqrt{5}$. 
From (44), the homoclinic bifurcation value of the damping coefficient $\mu_{0}$ is

$$
\begin{aligned}
\mu_{0} & =\frac{-1}{120} \\
& \cdot \frac{22625 \sqrt{6} \operatorname{arctanh}(3 \sqrt{3} /(4 \sqrt{2}+\sqrt{5}))-44478}{20 \sqrt{6} \operatorname{arctanh}(3 \sqrt{3} /(4 \sqrt{2}+\sqrt{5}))-63} \\
& =-0.241 .
\end{aligned}
$$

Then from (46) and (47), the homoclinic solution can be obtained as below,

$$
\begin{aligned}
& x=\frac{-9}{8+\sqrt{10} \cosh \tau}+\frac{1}{2}, \\
& \dot{x}=\frac{45 \sqrt{5}\left[\sqrt{3 / 2}+\omega_{1}(\tau)\right] \sinh \tau}{\sqrt{2}(4 \sqrt{10}+5 \cosh \tau)^{2}},
\end{aligned}
$$

where

$$
\begin{aligned}
& \omega_{1}(\tau)=-250 \sqrt{3} \sinh ^{3} \tau(2032 \sqrt{2}+392 \sqrt{5} \cosh \tau \\
& \left.+49 \sqrt{2} \cosh ^{2} \tau\right) \operatorname{arctanh}\left(\frac{3 \sqrt{3}}{4 \sqrt{2}+\sqrt{5}}\right) \\
& +98 \sqrt{3}(8192 \sqrt{5}+25600 \sqrt{2} \cosh \tau \\
& +12800 \sqrt{5} \cosh ^{2} \tau+8000 \sqrt{2} \cosh ^{3} \tau \\
& \left.+1000 \sqrt{5} \cosh ^{4} \tau+125 \sqrt{2} \cosh ^{5} \tau\right) \\
& +\operatorname{arctanh}\left(\frac{3 \sqrt{3}}{4 \sqrt{2}+\sqrt{5}} \tanh \frac{\tau}{2}\right)+63 \sinh \tau \\
& +(-39400 \\
& -14238 \sqrt{10} \cosh ^{2}-2040 \cosh ^{2} \tau \\
& \left.+175 \sqrt{10} \cosh ^{3} \tau\right) .
\end{aligned}
$$

The time history diagrams and the phase portraits of the solutions by different methods are shown in Figures 3 and 4, respectively. It can be seen from the figures that the present method still shows preference in the comparison. By the numerical method, the homoclinic bifurcation point can be found at $\mu_{c}=-0.239$, which are very close to those predicted by the present method.

\section{Application to the Chaos Prediction of the Nonautonomous Helmholtz-Duffing Oscillator}

Consider the nonautonomous Helmholtz-Duffing equation

$$
\ddot{x}+c_{1} x+c_{2} x^{2}+c_{3} x^{3}=\varepsilon\left(\mu_{0} \dot{x}+F \cos \Omega t\right),
$$

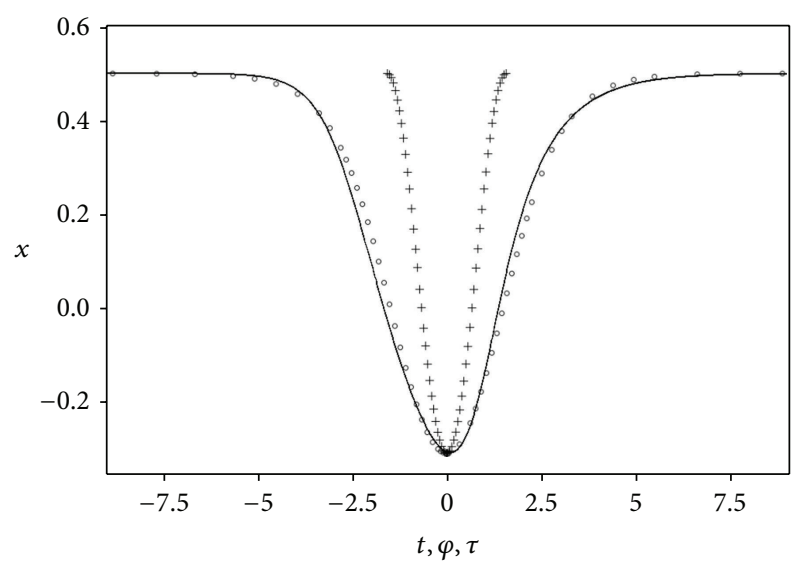

FIGURE 3: Time histories for homoclinic solutions of (48): - AUTO numerical method $[30,31] ; \bigcirc \bigcirc \bigcirc$ present method; +++ generalized harmonic function perturbation method [20].

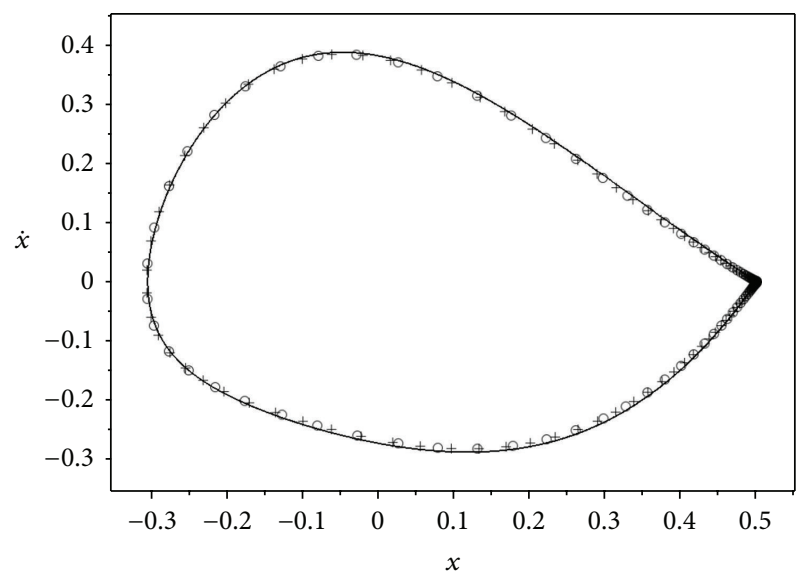

FIgURE 4: Phase portrait for homoclinic solutions of (48): - AUTO numerical method $[30,31] ; \bigcirc \bigcirc \bigcirc$ present method; +++ generalized harmonic function perturbation method [20].

where $F \cos \Omega t$ is the external harmonic excitation with the amplitude $F$ and the frequency $\Omega$. According to the Melnikov method $[1,2]$, the Melnikov function of $(52)$ can be written as

$$
\begin{aligned}
& M(\theta)=\int_{-\infty}^{+\infty}\left[\dot{x}_{0}, c_{1} x_{0}+c_{2} x_{0}^{2}+c_{3} x_{0}^{3}\right]^{\mathrm{T}} \\
& \wedge\left[0, \mu_{0} \dot{x}_{0}+F \sin \Omega(t+\theta)\right]^{\mathrm{T}} d \theta \\
& \quad=\mu_{0} \int_{-\infty}^{+\infty} \dot{x}_{0}^{2} d t-F \sin (\Omega \theta) \int_{-\infty}^{+\infty} \dot{x}_{0} \sin (\Omega t) d t
\end{aligned}
$$

where $x_{0}$ is the solution presented in (6) and $\wedge$ denotes the vector cross product. Then the chaotic response of the system may occur if there exists a simple zero point of $M(\theta)$. Thus, we substitute (7) into (53), and let $M(\theta)=0$; the latter yields

$$
M(\theta)=F \sin \Omega \theta+\frac{I}{J}=0,
$$


where

$$
\begin{aligned}
I= & \frac{a_{0}^{2} \omega_{0}}{\left(b^{2}-1\right)^{2}}\left[\frac{-b \mu_{0}}{\sqrt{b^{2}-1}}\right. \\
& \cdot \operatorname{arctanh}\left(\sqrt{\frac{b-1}{b+1}} \tanh \frac{\omega_{0} t}{2}\right) \\
& \left.+\frac{\sinh \omega_{0} t}{6\left(\cosh \omega_{0} t+b\right)^{3}} \mu_{0} \sum_{i=0}^{2} A_{i} \cosh ^{i} \omega_{0} t\right], \\
J= & a_{0} \omega_{0} \int_{-\infty}^{+\infty} \frac{\sinh \omega_{0} t \sin \Omega t}{\left(b+\cosh \omega_{0} t\right)^{2}} d t .
\end{aligned}
$$

Therefore, from (54) it can be seen that $M(\theta)$ possesses simple zero points if

$$
F>F_{c}=\left|\frac{I}{J}\right|,
$$

by which the onset of chaos can be evaluated with the threshold value $F_{c}$.

Furthermore, if we rewrite (52) as

$$
\ddot{x}+c_{1} x+c_{2} x^{2}+c_{3} x^{3}-\varepsilon \mu_{0} \dot{x}=\varepsilon F \cos \Omega t,
$$

and consider using the homoclinic solution expressed in (47) for Melnikov function, then we can derive

$$
\begin{aligned}
M(\theta)= & \int_{-\infty}^{+\infty}\left[\dot{x}_{0}, c_{1} x_{0}+c_{2} x_{0}^{2}+c_{3} x_{0}^{3}\right]^{\mathrm{T}} \\
& \wedge[0, F \sin \Omega(t+\theta)]^{\mathrm{T}} d \theta \\
= & F \int_{-\infty}^{+\infty} \dot{x} \cos \Omega(t+\theta) d t
\end{aligned}
$$

instead. Note that $\dot{x}$ is odd function of $t$. For any real value of $F$, there exists $\theta=2 k \pi$, where $k$ can be any integer number, to satisfy $M(\theta)=0$. That means the condition for chaos prediction becomes less restricted than that presented with the exact homoclinic solution of conservative system. Comparing (58) and (53) we can see that the difference is caused by omitting of the damping term $\mu_{0} \dot{x}$ in the Melnikov function calculation. In essence, the Melnikov method is based on approximation theory and, according to the method, it should be better to arrange all the perturbation terms at the right hand side of the equation to take them into account in the Melnikov function calculation. In other words, if we get the homoclinic solutions with perturbation method firstly and then implement the Melnikov function, we probably get a less restricted condition, because actually we conduct the perturbation approximations twice.

It is worth pointing out that as the present solutions by hyperbolic functions are explicit in respect to the real time $t$, they are more applicable to the Melnikov method for chaos prediction than those derived implicitly by generalized harmonic functions $[12,13,19,20]$. In particular, it should also

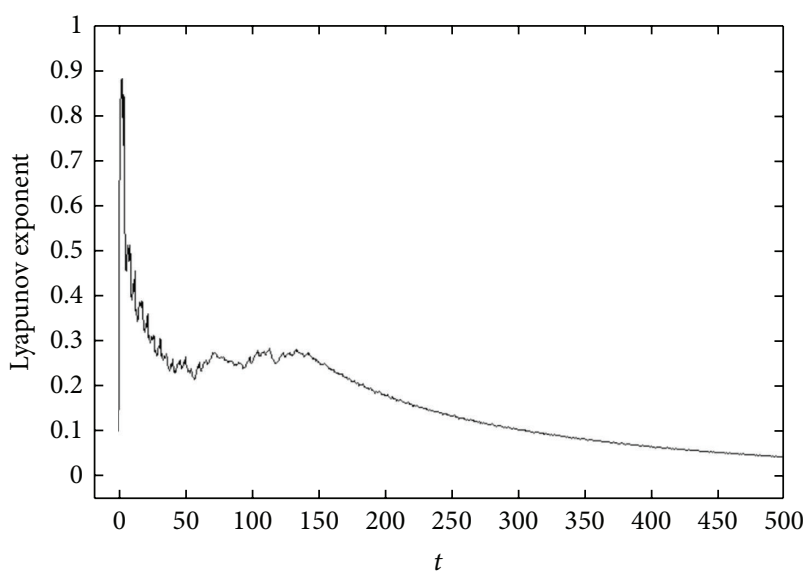

FIGURE 5: Lyapunov exponent diagram of (59) with $F=3$.

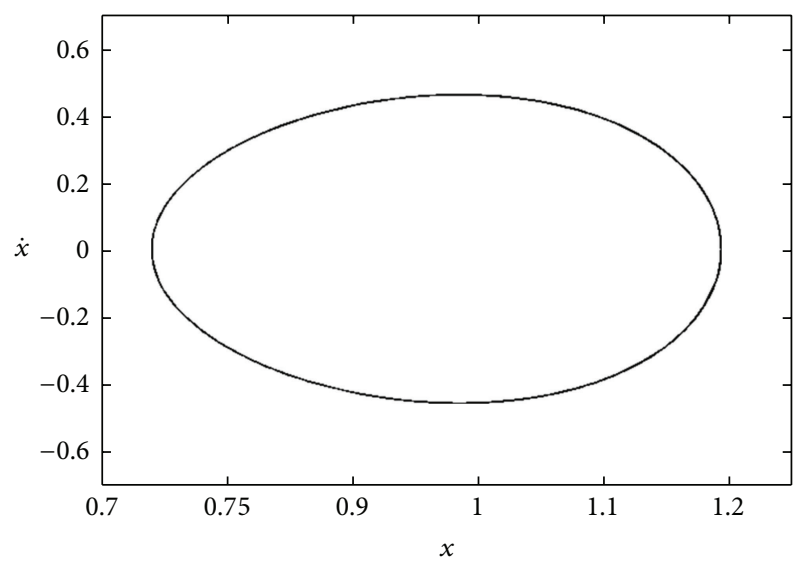

FIgURE 6: Limit cycle of (59) with $F=3$.

be pointed out that the Melnikov method is only regarded as one of the conditions for chaotic prediction. At present, a chaotic motion should still be evaluated more thoroughly with qualitative theory and numerical method. Below are two examples which satisfy the Melnikov condition but with different characters of chaotic motions.

Example 3. Consider the equation

$$
\ddot{x}-x-x^{2}+2 x^{3}=0.1(-\dot{x}+F \cos 2 t),
$$

which is the case of (52) with $c_{1}=-1, c_{2}=-1, c_{3}=2, \varepsilon=0.1$, $\mu_{0}=-1$, and $\Omega=2$. From (17), (55), and (56) we can derive that $F_{c}=1.43$ when $a_{0}=0.94868$ and $F_{c}=1.11$ when $a_{0}=-0.94868$. Therefore it can be estimated that chaotic motion may happen if $F>1.43$. The numerical results of homoclinic bifurcation by AUTO numerical method [30, 31] show that, with $F=3$, the Lyapunov exponent value shows chaos behavior from $t=0$ to about $t=145$ and then converts to 0 gradually. That means the chaotic motion possesses dissipative chaos property. The Lyapunov exponent diagram is shown in Figure 5. The Lyapunov exponent value converts to less than 0.01 after $t=1000$. The phase portrait of the system after $t=1000$ is shown in Figure 6, which shows that the motion converts to a limit cycle. 


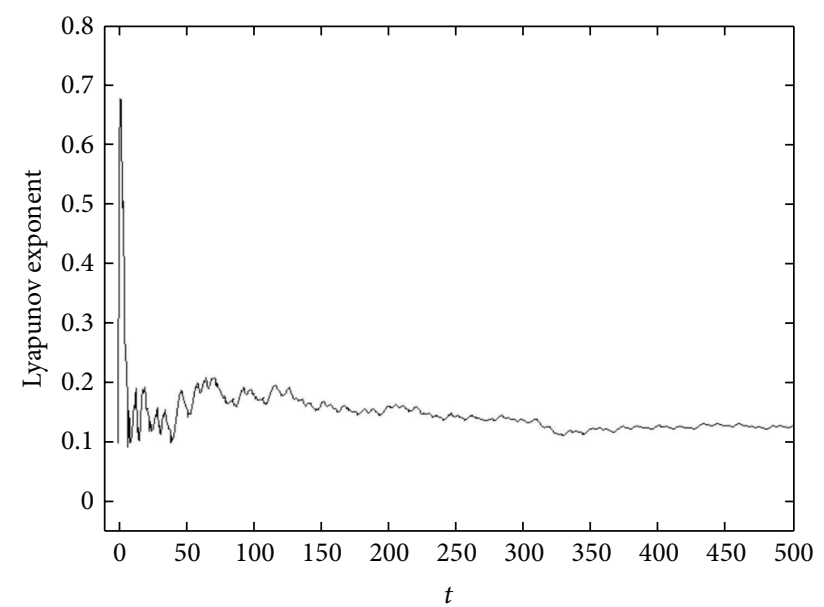

Figure 7: Lyapunov exponent diagram of (60) with $F=2.90$.

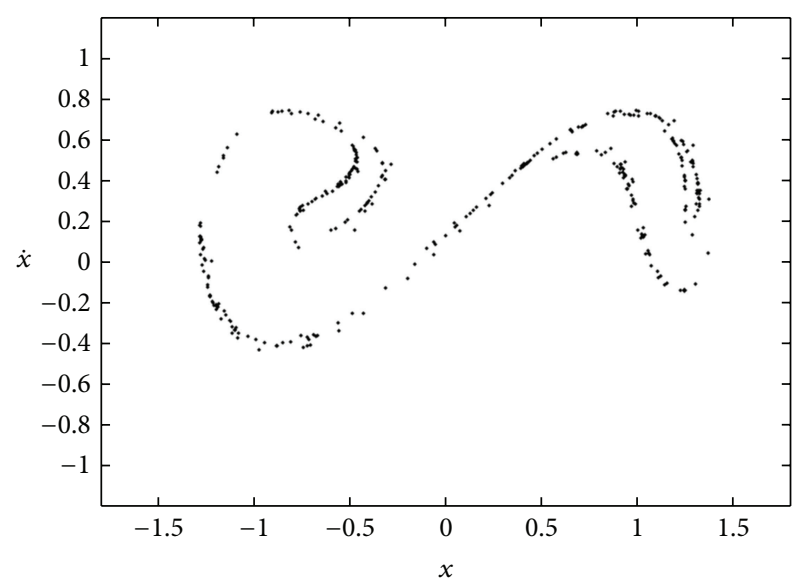

Figure 8: Strange attractor of (60) with $F=2.90$.
Example 4. Consider the equation

$$
\ddot{x}-x-0.05 x^{2}+x^{3}=0.1(-2.5 \dot{x}+F \cos t),
$$

which is the case of (52) with $c_{1}=-1, c_{2}=-0.05, c_{3}=1$, $\varepsilon=0.1, \mu_{0}=-2.5$, and $\Omega=1$. From (17), (55), and (56) we can derive that $F_{c}=1.94$ when $a_{0}=1.4138$ and $F_{c}=1.82$ when $a_{0}=-1.4138$. Therefore it can be estimated that chaotic motion may happen if $F>1.94$. The numerical results of homoclinic bifurcation by AUTO numerical method [30, 31] show that the Lyapunov exponent value stays more than 0 when $F=2.90$. The Lyapunov exponent diagram is shown in Figure 7. The Poincaré projection of the system from $t=$ 500 to $t=5000$ is shown in Figure 8. In the figure, the fractal character of a strange attractor can be observed, which supports the prediction of chaotic motion.

\section{Conclusions}

The present procedures are efficient for constructing homoclinic solutions of the Helmholtz-Duffing oscillator. The exact and explicit homoclinic solution of the undamped Helmholtz-Duffing oscillator is derived by a hyperbolic function balance procedure. The homoclinic solution of the self-excited system is then obtained by the extension of the hyperbolic perturbation procedure. The application to the chaos prediction of the nonautonomous Helmholtz-Duffing oscillator can also be conducted.

\section{Appendix}

Consider

$$
\begin{aligned}
& \beta=\frac{-4 b^{3} u-4 a_{0} b^{2}+4 b u-a_{0}}{4\left(b^{2}-1\right)} \\
& \gamma=\frac{-4 b^{5} u^{2}-8 a_{0} b^{4} u-4 a_{0}^{2} b^{3}+8 b^{3} u^{2}+6 a_{0} b^{2} u-3 a_{0}^{2} b-4 b u^{2}+2 a_{0} u}{4\left(b^{2}-1\right)^{2}} \\
& A_{0}=5 b^{2}-2 \\
& A_{1}=3 b\left(b^{2}+1\right) \\
& A_{2}=b^{2}+2 \\
& B_{0}=20 b^{5} u+28 a_{0} b^{4}-28 b^{3} u-19 a_{0} b^{2}+8 b u+6 a_{0}, \\
& B_{1}=12 b^{6} u+12 a_{0} b^{5}+20 b^{4} u+37 a_{0} b^{3}-40 b^{2} u-4 a_{0} b, \\
& B_{2}=16 b^{5} u+8 a_{0} b^{4}+4 b^{3} u+40 a_{0} b^{2}-20 b u-3 a_{0}, \\
& B_{3}=4 b^{4} u+2 a_{0} b^{3}+4 b^{2} u+13 a_{0} b-8 u \\
& D_{0}=100 b^{8} u^{2}+280 a_{0} b^{7} u+180 a_{0}^{2} b^{6}-240 b^{6} u^{2}-470 a_{0} b^{5} u-149 a_{0}^{2} b^{4}+180 b^{4} u^{2}+250 a_{0} b^{3} u+98 a_{0}^{2} b^{2}-40 b^{2} u^{2} \\
& -60 a_{0} b u-24 a_{0}^{2},
\end{aligned}
$$




$$
\begin{aligned}
D_{1} & =60 b^{9} u^{2}+120 a_{0} b^{8} u+60 a_{0}^{2} b^{7}+140 b^{7} u^{2}+530 a_{0} b^{6} u+375 a_{0}^{2} b^{5}-540 b^{5} u^{2}-880 a_{0} b^{4} u-25 a_{0}^{2} b^{3}+420 b^{3} u^{2} \\
& +290 a_{0} b^{2} u+10 a_{0}^{2} b-80 b u^{2}-60 a_{0} u, \\
D_{2}= & 140 b^{8} u^{2}+200 a_{0} b^{7} u+60 a_{0}^{2} b^{6}-20 b^{6} u^{2}+570 a_{0} b^{5} u+563 a_{0}^{2} b^{4}-420 b^{4} u^{2}-840 a_{0} b^{3} u-a_{0}^{2} b^{2}+340 b^{2} u^{2} \\
\quad+ & 70 a_{0} b u+8 a_{0}^{2}-40 u^{2}, \\
D_{3}= & 100 b^{7} u^{2}+100 a_{0} b^{6} u+30 a_{0}^{2} b^{5}-60 b^{5} u^{2}+430 a_{0} b^{4} u+355 a_{0}^{2} b^{3}-180 b^{3} u^{2}-560 a_{0} b^{2} u+35 a_{0}^{2} b+140 b u^{2}+30 a_{0} u, \\
D_{4}= & 20 b^{6} u^{2}+20 a_{0} b^{5} u+6 a_{0}^{2} b^{4}+110 a_{0} b^{3} u+83 a_{0}^{2} b^{2}-60 b^{2} u^{2}-130 a_{0} b u+16 a_{0}^{2}+40 u^{2} .
\end{aligned}
$$

\section{Conflict of Interests}

The authors hereby declare that there is no conflict of interests regarding the publication of this paper.

\section{Acknowledgments}

The financial supports from the National Basic Research Program of China (2011CB013606), the National Natural Science Foundations of China (91315301-07, 11102045), and the National Plan for Science and Technology Support of China (2012BAJ07B02) are gratefully acknowledged.

\section{References}

[1] A. H. Nayfeh and B. Balachandran, Applied Nonlinear Dynamics: Analytical, Computational, and Experimental Method, Wiley-Interscience, New York, NY, USA, 1995.

[2] J. Guckenheimer and P. Holmes, Nonlinear Oscillations, Dynamical Systems, and Bifurcation of Vector Field, Springer, New York, NY, USA, 2002.

[3] F. C. Moon, J. Cusumano, and P. J. Holmes, "Evidence for homoclinic orbits as a precursor to chaos in a magnetic pendulum," Physica D: Nonlinear Phenomena, vol. 24, no. 1-3, pp. 383-390, 1987.

[4] A. L. Katz and E. H. Dowell, "From single well chaos to cross well chaos: a detailed explanation in terms of manifold intersections," International Journal of Bifurcation and Chaos, vol. 4, no. 4, pp. 933-941, 1994.

[5] J. J. Feng, Q. C. Zhang, and W. Wang, "Chaos of several typical asymmetric systems," Chaos, Solitons \& Fractals, vol. 45, no. 7, pp. 950-958, 2012.

[6] S. Lenci, G. Menditto, and A. M. Tarantino, "Homoclinic and heteroclinic bifurcations in the non-linear dynamics of a beam resting on an elastic substrate," International Journal of NonLinear Mechanics, vol. 34, no. 4, pp. 615-632, 1999.

[7] S. Lenci and G. Rega, "Control of the homoclinic bifurcation in buckled beams: infinite dimensional vs reduced order modeling," International Journal of Non-Linear Mechanics, vol. 43, no. 6, pp. 474-489, 2008.

[8] J. H. Merkin and D. J. Needham, "On infinite period bifurcations with an application to roll waves," Acta Mechanica, vol. 60, no. 1-2, pp. 1-16, 1986.
[9] I. M. Uzunov, "Description of the suppression of the soliton selffrequency shift by bandwidth-limited amplification," Physical Review E, vol. 82, Article ID 066603, 8 pages, 2010.

[10] I. M. Uzunov and T. N. Arabadzhiev, "Suppression of the soliton self-frequency shift and compression in the presence of bandwidth-limited amplification," Physical Review E, vol. 84, no. 2, Article ID 026607, 9 pages, 2011.

[11] Q. Bi, "Singular solitary waves associated with homoclinic orbits," Physics Letters A, vol. 352, no. 3, pp. 227-232, 2006.

[12] Z. Xu, H. S. Y. Chan, and K. W. Chung, "Separatrices and limit cycles of strongly nonlinear oscillators by the perturbationincremental method," Nonlinear Dynamics, vol. 11, no. 3, pp. 213-233, 1996.

[13] H. S. Y. Chan, K. W. Chung, and Z. Xu, "Stability and bifurcations of limit cycles by the perturbation-incremental method," Journal of Sound and Vibration, vol. 206, no. 4, pp. 589-604, 1997.

[14] M. Belhaq, B. Fiedler, and F. Lakrad, "Homoclinic connections in strongly self-excited nonlinear oscillators: the Melnikov function and the elliptic Lindstedt-Poincaré method," Nonlinear Dynamics, vol. 23, no. 1, pp. 67-86, 2000.

[15] Y. V. Mikhlin and G. V. Manucharyan, "Construction of homoclinic and heteroclinic trajectories in mechanical systems with several equilibrium positions," Chaos, Solitons and Fractals, vol. 16, no. 2, pp. 299-309, 2003.

[16] G. V. Manucharyan and Y. V. Mikhlin, "The construction of homo- and heteroclinic orbits in non-linear systems," Journal of Applied Mathematics and Mechanics, vol. 69, pp. 42-52, 2005.

[17] Y. Y. Chen and S. H. Chen, "Homoclinic and heteroclinic solutions of cubic strongly nonlinear autonomous oscillators by the hyperbolic perturbation method," Nonlinear Dynamics, vol. 58, no. 1-2, pp. 417-429, 2009.

[18] S. H. Chen, Y. Y. Chen, and K. Y. Sze, "A hyperbolic perturbation method for determining homoclinic solution of certain strongly nonlinear autonomous oscillators," Journal of Sound and Vibration, vol. 322, no. 1-2, pp. 381-392, 2009.

[19] Y. Y. Cao, K. W. Chung, and J. Xu, "A novel construction of homoclinic and heteroclinic orbits in nonlinear oscillators by a perturbation-incremental method," Nonlinear Dynamics, vol. 64, no. 3, pp. 221-236, 2011.

[20] Z. B. Li, J. S. Tang, and P. Cai, "A generalized harmonic function perturbation method for determining limit cycles and homoclinic orbits of Helmholtz-Duffing oscillator," Journal of Sound and Vibration, vol. 332, no. 21, pp. 5508-5522, 2013.

[21] S. H. Chen and Y. K. Cheung, "A modified Lindstedt-Poincaré method for a strongly nonlinear system with quadratic and 
cubic nonlinearities," Shock and Vibration, vol. 3, no. 4, pp. 279285, 1996.

[22] A. Y. T. Leung and Z. Guo, "Homotopy perturbation for conservative Helmholtz-Duffing oscillators," Journal of Sound and Vibration, vol. 325, no. 1-2, pp. 287-296, 2009.

[23] Z. Guo and A. Y. T. Leung, “The iterative homotopy harmonic balance method for conservative Helmholtz-Duffing oscillators," Applied Mathematics and Computation, vol. 215, no. 9, pp. 3163-3169, 2010.

[24] H. Askari, Z. Saadatnia, D. Younesian, A. Yildirim, and M. Kalami-Yazdi, "Approximate periodic solutions for the Helmholtz-Duffing equation," Computers and Mathematics with Applications, vol. 62, no. 10, pp. 3894-3901, 2011.

[25] A. Elías-Zúñiga, "Exact solution of the quadratic mixed-parity Helmholtz-Duffing oscillator," Applied Mathematics and Computation, vol. 218, no. 14, pp. 7590-7594, 2012.

[26] A. Elías-Zúñiga, "Analytical solution of the damped HelmholtzDuffing equation," Applied Mathematics Letters, vol. 25, no. 12, pp. 2349-2353, 2012.

[27] K.-E. Thylwe, "Exact quenching phenomenon of undamped driven Helmholtz and Duffing oscillators," Journal of Sound and Vibration, vol. 161, no. 2, pp. 203-211, 1993.

[28] Y. Y. Lee, X. Guo, and E. W. M. Lee, "Effect of the large amplitude vibration of a finite flexible micro-perforated panel absorber on sound absorption," International Journal of Nonlinear Sciences and Numerical Simulation, vol. 8, no. 1, pp. 41-44, 2007.

[29] S. K. Lai, J. Harrington, Y. Xiang, and K. W. Chow, "Accurate analytical perturbation approach for large amplitude vibration of functionally graded beams," International Journal of NonLinear Mechanics, vol. 47, no. 5, pp. 473-480, 2012.

[30] E. J. Doedel, H. B. Keller, and J.-P. Kernévez, "Numerical analysis and control of bifurcation problems (I): bifurcation in finite dimensions," International Journal of Bifurcation and Chaos, vol. 1, no. 3, pp. 493-520, 1991.

[31] E. J. Doedel, H. B. Keller, and J.-P. Kernévez, "Numerical analysis and control of bifurcation problems. (II): bifurcation in infinite dimensions," International Journal of Bifurcation and Chaos in Applied Sciences and Engineering, vol. 1, no. 4, pp. 745772, 1991. 


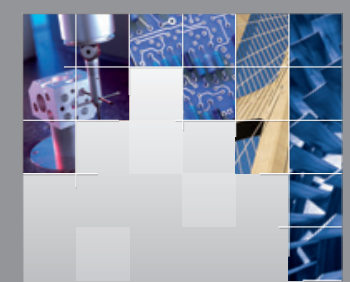

\section{Enfincering}
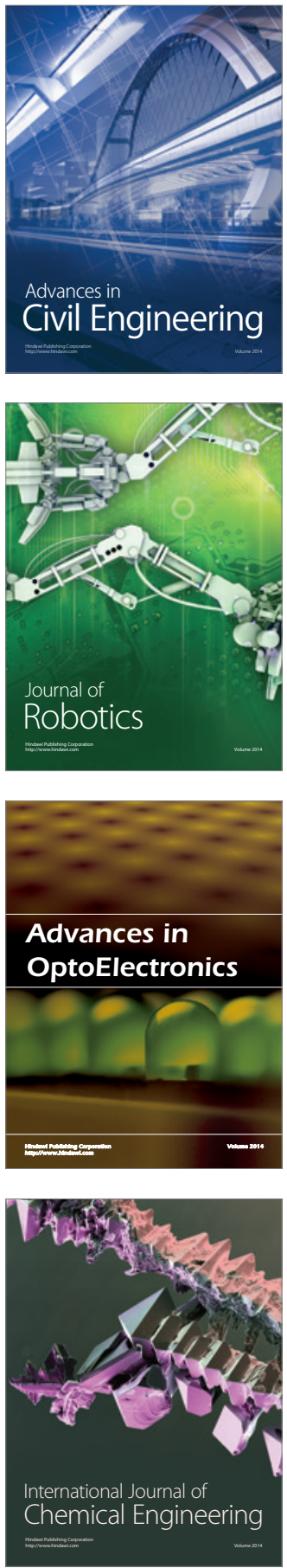

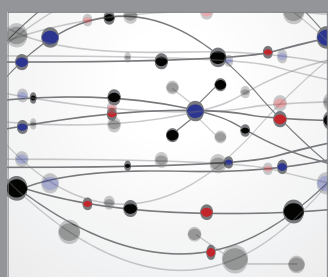

The Scientific World Journal

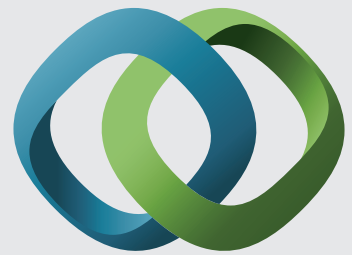

\section{Hindawi}

Submit your manuscripts at

http://www.hindawi.com
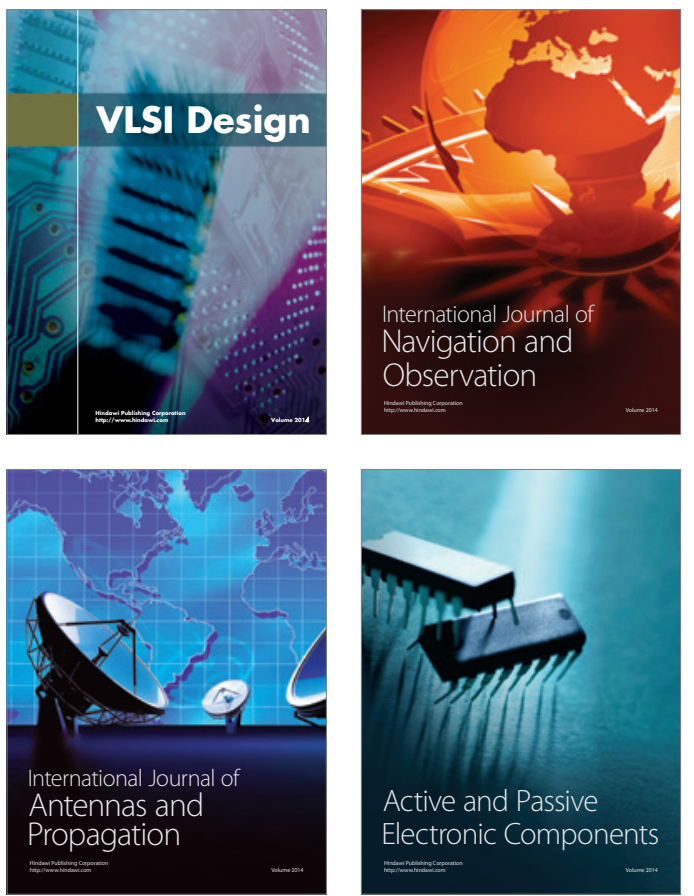
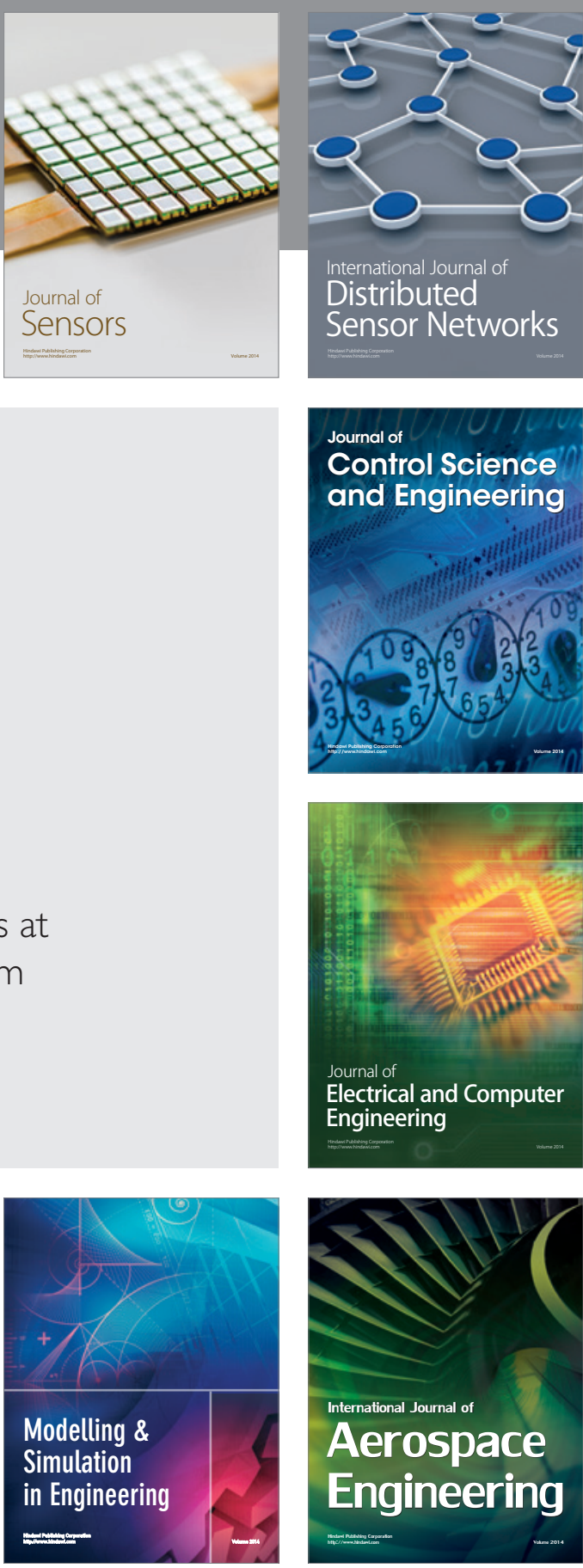

International Journal of

Distributed

Sensor Networks

Journal of

Control Science

and Engineering
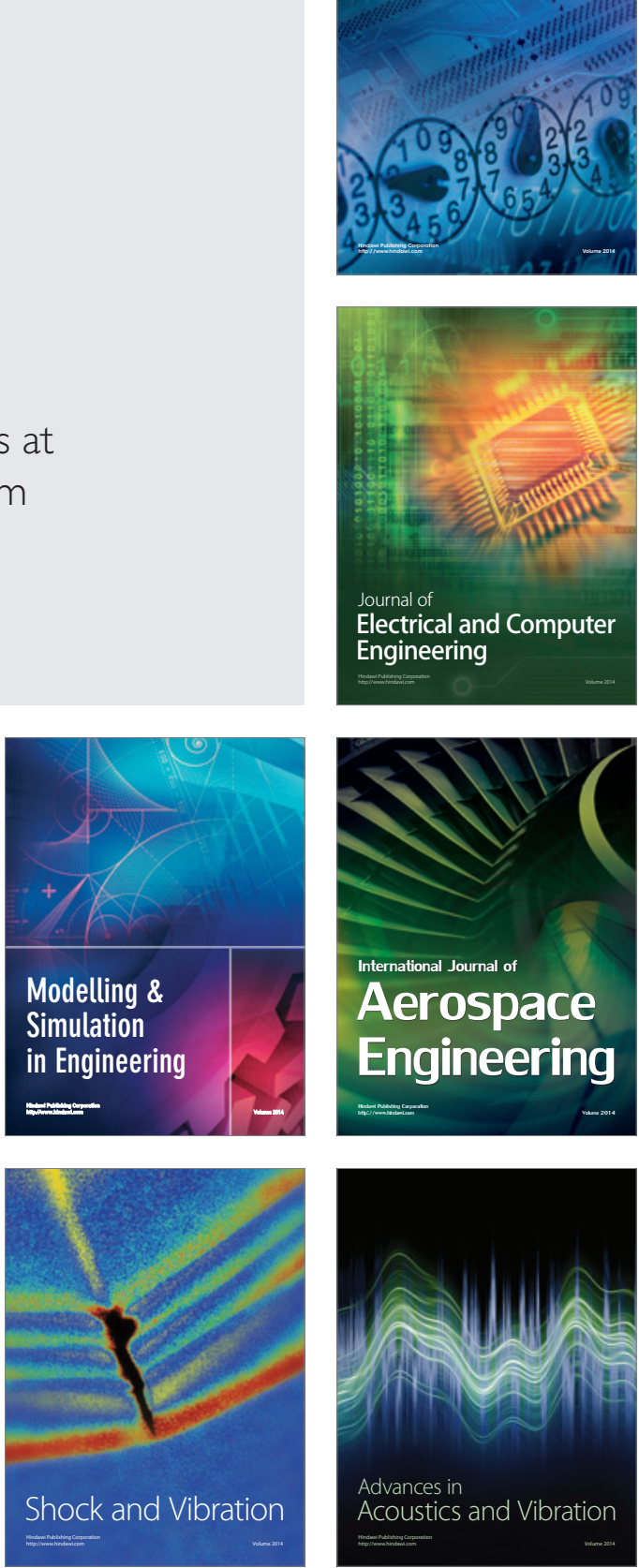